\title{
El Che Guevara y el desarrollo de los recursos humanos en Cuba
}

\author{
Tirso W. Sáenz
}

\section{Resumen}

En la vida y obra de Ernesto Che Guevara (1928-1967) hay aspectos cuya indagación debe contribuir, por ser menos conocidos, a resaltar su destacado papel en la construcción del socialismo en Cuba, desde su posición como Ministro de Industrias. En esta función desempeñó un importante papel en el proceso de industrialización cubano y, vinculado al mismo, en la promoción y el desarrollo de los recursos humanos en esta esfera. Todo lo anterior se sustentaba en un profundo desarrollo de la conciencia del hombre y del papel de éste como actor central y objetivo principal en la construcción de la nueva sociedad socialista. En el presente artículo se muestran las concepciones, las prioridades establecidas y las acciones consecuentes tomadas por el Che en ese sentido.

\section{Palabras clave}

Che Guevara, recursos humanos, capacitación, socialismo, revolución cubana, Ministerio de Industrias de Cuba

\section{Introducción}

...no hay nada que eduque más a un hombre honrado que el vivir dentro de una revolución. Che Guevara

Existe una corriente que trata de presentar al Ernesto Che Guevara como un mito, un soñador, casi un santo, algo inalcanzable e irrepetible, que dio su vida por un noble ideal, pero que ya no tiene presente ni futuro. Al mismo tiempo, esa corriente deliberadamente ignora su labor creadora como constructor de una nueva sociedad y a negar y ocultar la vigencia de su pensamiento y de su acción en los momentos actuales.

Mas, en la vida y obra del Che, hay aspectos cuya indagación debe contribuir, por ser menos conocidos que otros, a una mejor comprensión de su multifacética personalidad. Uno de esos aspectos es, precisamente, el concerniente a su participación directa en la construcción de la base técnico-material del socialismo en Cuba y, en particular, a su gestión como Ministro de Industrias. En esta función desempeñó un importante papel en el proceso de industrialización cubano y, vinculado al mismo, en la promoción y organización del progreso científico y tecnológico y en particular el desarrollo de los recursos humanos en este sector. Todo lo anterior se sustentaba en un profundo desarrollo de la conciencia del hombre y del papel de éste como actor central y objetivo principal en la construcción de la nueva sociedad socialista.

(*) Ex-Vice-ministro de Industrias de la República de Cuba y ex-Vice-presidente da Academia de Ciencias de Cuba. Dirección electrónica: tirsowsaenz@gmail.com 
Por otra parte, el Che comprendía que el factor político no se basaba exclusivamente en el hecho de haber tenido una participación en el proceso revolucionario, o hacer profesión explícita de fe en la Revolución. A este elemento tenían que unirse elementos esenciales tales como liderazgo, sensibilidad humana, austeridad y modestia, entre otros, sin los cuales, tampoco se podía tener responsabilidades en diferentes niveles. El oportunismo, la arrogancia, los métodos de ordeno y mando no tenían cabida, mucho menos, los que trataban de obtener beneficios personales en el cargo.

\section{El punto de partida}

En vísperas de la Revolución, en 1958, había en Cuba casi un millón de analfabetos y sólo recibían educación primaria setecientos diecisiete mil alumnos, es decir, la mitad de los niños en edad de asistir a la escuela no lo hacían. La educación técnica de nivel medio era muy escasa; se concentraba en las llamadas escuelas de Artes y Oficios. La educación superior no estaba preparada para satisfacer las demandas que reclamaba el desarrollo, tanto en el orden de la cantidad de especialistas que se debían formar, como en el de los perfiles necesarios y la calidad requerida. En la estructura de la matrícula, las humanidades alcanzaban un $23 \%$ y la tecnología sólo un $11 \%$. La formación se caracterizaba por ser en gran parte teórica, alejada de la práctica y muy desvinculada de las realidades del país (Fernández, 1975).

La recuperación por parte del Gobierno Revolucionario de establecimientos industriales pertenecientes a malversadores, latifundistas y propietarios burgueses cubanos vinculados con la tiranía de Fulgencio Batista, quienes huyeron del país en los primeros meses después del triunfo de la Revolución, así como las ulteriores nacionalizaciones que culminaron el 13 de octubre de 1960, llevaron a la creación del Ministerio de Industrias (MININD), donde se concentró la casi totalidad de la producción industrial. Para dirigirlo se designó al comandante Ernesto Che Guevara. Ya en 1961, la parte fundamental de los medios de producción del país en la industria y otros sectores habían pasado a la propiedad del Estado.

En el MININD, el enfoque para los trabajos del desarrollo industrial y científicotecnológico fue integral. No solo abarcaba los aspectos de la investigación y el desarroIlo, sino los problemas de la transferencia de tecnología, la organización racional del trabajo, la información científico-técnica, la normalización, la metrología y el control de la calidad, la modernización de los procesos administrativos y, especialmente, la calificación de los recursos humanos en todos los niveles entre otros. Se trataba, pues, de que todas estas actividades se interconectaran y se vincularan estrechamente con los problemas actuales y perspectivo de la producción.

Tomando en cuenta un número de factores, como el grado de dependencia de suministros de equipamiento, maquinarias, materiales, piezas de repuesto y de tecnologías respecto a los Estados Unidos, el Bloqueo imperialista había impuesto al país que cortaba el suministro de materias primas y demás insumos; el carácter artesanal de la mayoría de los centros manufactureros y, por último, la escasez de personal técnico. Cabe destacar que todo ello fue agravado por el abandono del país por la mayor parte de él; el bajo nivel técnico y cultural en general de los trabajadores y de muchos de quienes tuvieron que asumir la dirección de las fábricas; el volumen, complejidad y heterogeneidad del conjunto de las industrias agrupadas en el organismo; la inexperiencia en general de todo el aparato central del Ministerio y sus empresas en la con- 
ducción armónica y planificada de toda la industria; la débil y escasa infraestructura productiva y la falta de planes formalizados de desarrollo económico y social a mediano y largo plazos.

Mantener la producción en estas circunstancias era, por supuesto, una tarea que requería ingentes esfuerzos de dirección. Realizar esto, y al mismo tiempo, elaborar sobre la marcha una estrategia que incluyese necesariamente la formación priorizada de recursos humanos y que permitiera tomar medidas tempranas que asegurasen el futuro con independencia de las correcciones por aproximaciones sucesivas que iba marcando la ejecución de toda estrategia. Queda confirmada, por tanto, la visión de la Revolución Cubana, de Fidel Castro (1926-2016) y, en particular del Che Guevara, en cuanto a la voluntad de avanzar y vencer las innumerables dificultades presentes y al mismo tiempo progresar en el desarrollo social, ideológico científico y tecnológico.

El pensamiento del Che se caracterizaba por su integralidad. Las concepciones políticas, económicas, sociales de su pensamiento están estrechamente interrelacionadas y se llevaron a la práctica de manera consecuente. Su acción en este sentido derivaba del criterio, resueltamente planteado por él en El socialismo y el hombre en Cuba (1970), texto dirigido a Carlos Quijano que posteriormente fue publicado en la revista Marcha (1965, Montevideo), de que la formación del hombre nuevo y el desarrollo de la técnica eran los dos pilares para la construcción del socialismo y el comunismo.

Por otra parte, destacaba la necesidad de comprender la importancia en el mundo contemporáneo de estudiar, concebir y utilizar buenas técnicas de dirección y la urgencia de que nuestra naciente sociedad socialista asimilara rápidamente estos instrumentos.

Destacó, además, que los dirigentes de la producción tuvieran una adecuada formación política, económica y tecnológica. De ello derivó, la necesidad de elevar los conocimientos científicos de dirección como condición indispensable para hacer de estos dirigentes verdaderos cuadros de dirección económica a la altura de los requerimientos de la sociedad. Che supo descubrir los resultados administrativos alcanzados en nuestro país por las empresas monopolistas norteamericanas, haciendo patente la necesidad de apropiarse de estas experiencias y aplicarlas en nuestra administración (Guevara, 1977, p. 183). Los manuales de administración, la contabilidad y su control, la utilización de computadoras, la definición de atribuciones de órganos y dirigentes, fueron necesidades imperiosas de una correcta dirección económica y requisitos de conocimientos para nuestros dirigentes a todos los niveles.

\section{La capacitación: criterios básicos}

Para encarar el proceso de industrialización trazado se requerían ingentes esfuerzos dirigidos a la calificación acelerada de toda la fuerza laboral, pues de su capacidad y experiencia dependía decisivamente la realización del desarrollo industrial sobre bases científicas y tecnológicas. El Che resumía su concepción de la siguiente forma:

Cada día la industria moderna exige un adiestramiento mayor, una cultura técnica superior, los instrumentos y procesos productivos se tornan más complejos, más automatizados. La capacitación es necesaria, desde luego, para obtener un mayor rendimiento de las actuales instalaciones y recursos industriales, pero también es decisiva para planear la construcción de las nuevas fábricas. Muchas veces se dispone de las maquinarias y equipos para instalar una fábrica, pero se carece del conocimiento, de la experiencia para incorporarlos rápidamente a la producción. No es exagerado afirmar que la clave para poner en marcha un rápido proceso de industrialización y asegurar su éxito sostenido, está en la formación de una 
numerosa fuerza de trabajo altamente calificada: desde científicos a obreros especializados. (Guevara, 1966a, p. 95)

Además, indicaba los caminos en los primeros años de la Revolución para desarrollar los recursos humanos en la industria:

\begin{abstract}
La capacitación de los trabajadores activos se inicia en los centros de trabajo al primer nivel educacional: la eliminación de algunos restos de analfabetismo que quedan en los lugares más apartados, los cursos de seguimiento, después, los de superación obrera para aquellos que hayan alcanzado el tercer grado, los cursos de Mínimo Técnico para los obreros de mayor nivel, los de extensión para hacer subingenie$\operatorname{ros}^{1}$ a los obreros calificados, los cursos universitarios para todo tipo de profesional y, también los administrativos. La intención del Gobierno Revolucionario es convertir nuestro país en una gran escuela, donde el estudio y el éxito de los estudios sean uno de los factores fundamentales para el mejoramiento de la condición del individuo, tanto económicamente como en su ubicación dentro de la sociedad, de acuerdo con sus cualidades. (Guevara, 1966b, p. 153)
\end{abstract}

El Che consideraba que se estaban viviendo unos momentos de cambios tecnológicos tan fantásticos, que realmente era una obligación de todos los directores de empresas estudiar al máximo los avances tecnológicos y las novedades que iban surgiendo. Así, sobre la capacitación expresaba:

El complemento indispensable sin el cual todo el plan queda convertido en un sueño [es] la capacitación científica, tecnológica, técnica, de los cuadros de todos los niveles, con una dedicación especial al descubrimiento y desarrollo de futuros cuadros dirigentes (Guevara, 1972, p. 109).

\title{
Orientaciones y acciones consecuentes
}

Dada la importancia asignada a la capacitación, ésta fue una actividad altamente priorizada. El Che la visualizaba como una acción general, en todas las fábricas, empresas y en el propio Ministerio, siendo una responsabilidad de cada cuadro dirigente en su ámbito respectivo de actuación.

En sus Orientaciones para 1963, volvió a enfatizar que la capacitación era vital para el desarrollo de nuestra economía y, más aún, en esa etapa inicial de construcción del socialismo. Subrayó la necesidad de que todos los dirigentes estuvieran incorporados a círculos de estudio, que no tenían que ser necesariamente de tipo político; podían ser de tipo técnico o cultural. Consideraba imprescindible identificar y destacar la capacitación requerida para los planes perspectivos que estaban siendo elaborados por el MININD.

Un año más tarde, señaló que se habían obtenido avances sustanciales en esta tarea, aunque todavía no se había obtenido un éxito completo, ni en la capacitación masiva, ni en las específicas para las fábricas que serían construidas en los años siguientes, ni en la calificación de diferentes oficios comunes a muchas industrias. Criticó la poca atención que las empresas prestaban a la capacitación. Volvió a insistir en la necesidad del ejemplo de los dirigentes y técnicos en participar en círculos de estudios para aumentar su capacidad en materias afines a su trabajo, sin permitir que el trabajo cotidiano los hiciera olvidar la necesidad de capacitarse. Además, el Che insistía y volvía a insistir en el ejemplo de los dirigentes y en su visión perspectiva en cuanto a la capacitación.

(1) El término de subingenieros se usaba en esos momentos para referirse a técnicos de nivel medio. 
Fue el 1965 cuando señaló que se habían realizado avances notables respecto a la capacitación masiva y primaria de nuestros obreros, pero se presentaban debilidades en la capacitación de los niveles superiores, técnicos y administrativos. El ejemplo que los dirigentes deberían dar -como él había orientado- no se había cumplido a plenitud. Los directores de empresas y los administradores de fábricas no constituían el ejemplo deseado. Era necesario reflexionar y preparar cuidadosamente planes para formar los técnicos necesarios para un futuro más o menos inmediato.

El Ministerio se convirtió en una gran escuela. En cada fábrica se organizaron cursos de nivel primario y de Mínimo Técnico (Cursos sobre los conocimientos mínimos que se debían alcanzar para un desempeño adecuado en cada puesto de trabajo). Se abrieron escuelas como la de Administradores, la de Automatización y la de Dibujantes Mecánicos, entre otras. Además, se organizaron cursos especiales para la preparación del personal para las nuevas inversiones.

Alcanzar el sexto grado se convirtió en una obligación para todos los administradores de fábricas [En esos momentos se denominaban administradores de fábricas a los que actualmente se denominan directores]. Sería imposible, después de determinado momento, desarrollar exitosamente un proceso de industrialización con dirigentes de fábrica de baja escolaridad y calificación. Con tal motivo, en 1963, el Che dictó una resolución fijando el plazo de un año para que todos los administradores de fábricas alcanzaran el sexto grado. Los que no lo lograron fueron sustituidos de sus cargos, ya que era imposible después de determinado momento que pudieran desarrollarse exitosamente en un proceso de industrialización con dirigentes de base de muy baja calificación. Nótese que meta tan modesta estaba propuesta: jposeer la enseñanza primaria! Tal era la realidad de la época.

El Che se dio cuenta de que la meta de un año era imposible de alcanzar para una gran mayoría de administradores, quienes, al mismo tiempo, estaban haciendo un esfuerzo extraordinario para dirigir sus fábricas. Otra realidad de la época. Por tanto, amplió el plazo a un año más. Esta vez, los que no consiguieron alcanzar la meta en el nuevo plazo, fueron sustituidos. Sin embargo, en los casos de administradores sustituidos que habían demostrado un desempeño aceptable y perspectivas de desarrollo, se les envió a la Escuela de Administradores o a otros cursos de capacitación.

Una vez más, debe insistirse en la voluntad de desarrollar los recursos humanos a todos los niveles como necesidad imperiosa. También, como norma del Ministerio, hacer que determinadas tareas se cumplieran siguiendo el ejemplo de sus dirigentes. Pues bien, se trató, desde la creación del MININD, de estructurar sistemas que permitieran que los distintos técnicos y dirigentes de la producción aumentasen su capacidad en materias que le fueran afines, en la forma y con los métodos que resultasen más adecuados, pero sin permitir que el trabajo cotidiano haga olvidar totalmente la necesidad de su capacitación. Por otra parte, la capacitación debía adquirir características perspectivas para todo el aparato industrial a todos los niveles.

Uno de los logros más importantes alcanzados en el MININD fue haber impulsado una participación masiva de la clase obrera en la solución de problemas que diariamente afectaban la producción, como método de vital trascendencia política para el desarrollo y promoción de cuadros dirigentes: los Comités de Piezas de Repuesto y, por último, el Movimiento Construye tu Propia Maquinara que posteriormente se convirtió en la Asociación Nacional de Innovadores y Racionalizadores. Sobre ellos, el Che expre- 
só que eran... «el laboratorio experimental donde la clase obrera se prepara para la grandes tareas futuras de la conducción integral del país». (Guevara, 1972, p. 200)

\section{El ejemplo personal}

El Che había orientado que los dirigentes del MININD, a todos los niveles, debían ser ejemplos en el estudio. Él seguía el principio básico del ejemplo personal para impulsar las distintas tareas.

Primero, comenzó a tomar un curso de Matemática, cada jueves a las siete de la mañana. No cabe duda que esto representaba un esfuerzo grande, pues normalmente el Che se quedaba en el Ministerio hasta altas horas de la madrugada. Después organizó un curso de Matemática Superior, lunes a las nueve de la noche, en la que participamos varios dirigentes.

Cada miércoles a las nueve de la noche, tomaba, junto con otros dirigentes del Ministerio, un curso sobre El Capital de Karl Marx, impartido por un profesor, profundo conocedor de la obra marxista. Los que participaban en ese curso comentaban que las discusiones que se entablaban entre el Che y el profesor Anastasio Mansilla que se exilió de España tras la Guerra Civil (1936-1939) eran interesantísimas, las que muchas veces se prolongaban hasta altas horas de la madrugada. A pesar de eso, a la mañana siguiente se encontraría con el profesor para sus clases de Matemática.

Unas de las tareas fundamentales permanentes del MININD, era la disciplina financiera, para lo cual se requería que los dirigentes tuvieran una noción adecuada de Contabilidad. A esos fines, el Che le solicitó a un contador cubano, muy calificado, integrante de su grupo de asesores, para que le impartiera un curso sobre esta materia. Además de estos cursos organizados con cierta regularidad, el Che estudiaba personalmente y se asesoraba con diversos especialistas sobre los procesos tecnológicos más importantes de las empresas, sobre los caminos por los que avanzaba la tecnología en el mundo, así como las técnicas modernas de dirección. Por otra parte, leía asiduamente literatura, incluyendo poesía, de la cual gustaba particularmente.

Un lunes, a finales de 1964, al llegar al salón de reuniones para participar en el Consejo de Dirección, nos encontramos en la mesa, frente al asiento de cada uno, un libro pequeño: Programación Lineal. Le preguntamos al Che que para qué era ese libro. Respondió que la computación ya era una realidad en el mundo, que nosotros no teníamos la menor noción de eso y que era necesario y urgente aprender algunas de las herramientas relacionadas con esa disciplina. Por eso, a partir del siguiente lunes, a las 7 a.m., antes de las sesiones del Consejo de Dirección, comenzaríamos a recibir este curso. Cuando le preguntamos quién sería el profesor, respondió: Yo.

Así fue. No sólo se preparó para ser un buen profesor -y lo hizo bien-, sino que nos exigía que estudiásemos la materia previamente e hiciésemos, como estudiantes aplicados, las tareas que él nos señalaba.

Siguiendo este ejemplo, los viceministros y otros directores del Ministerio, comenzamos a tomar un curso de Economía Política con un economista del organismo. 


\section{La Dirección de Capacitación}

La Dirección de Capacitación fue creada tempranamente: desde la época del Departamento de Industrialización del Instituto Nacional de la Reforma Agraria (INRA), es decir, a los inicios del triunfo de la Revolución. Al crearse el MININD fue adscrita al Viceministerio para el Desarrollo Técnico. Su misión era la de asegurar metodológicamente, en coordinación con otros organismos, o con recursos propios, la calificación necesaria para todos los trabajadores del MININD. Era una tarea gigantesca por su magnitud y diversidad.

También demuestra, según nuestro entender, la visión perspectiva del Che el hecho de que la Dirección de Capacitación, al estructurarse el MININD, no fuera ubicada, como es frecuente, dentro del área de Recursos Humanos, de carácter más administrativo, sino dentro del Viceministerio para el Desarrollo Técnico.

Llegado a este punto, pasamos a recordar el siguiente ejemplo. Al nacionalizarse la industria, existían cientos de pequeñas unidades artesanales productoras de calzado, conformadas por núcleos pequeños de trabajadores de baja escolaridad. Un estudio inicial sobre la elevación de la productividad en este sector mostraba que, mediante simples medidas racionalizadoras, integrando las unidades pequeñas en otras mayores con cierto flujo elemental de la producción, existía la posibilidad de alcanzar incrementos sustanciales en este índice, pero generando un excedente de alrededor de cinco mil trabajadores en todo el país. Esta situación planteaba un posible problema social y político a la naciente Revolución, ya que la baja capacitación de dichos trabajadores no permitía que se les reubicara fácilmente en otros centros de producción y la política de la Revolución se dirigía a lograr el pleno empleo. Se imponía elaborar, por consiguiente, una fórmula que conjugara de manera creadora la posibilidad de lograr los tan necesarios incrementos de la productividad y los requerimientos de la lucha contra el desempleo. Este es uno de los círculos viciosos que los países subdesarrollados muchas veces no consiguen romper.

Refiriéndose a esta cuestión, el Che lo expresó con las siguientes palabras:

...todo obrero que está demás en una fábrica es un desocupado social (...) ¿sacarlos, para qué? Para botarlos no; para que vayan a escuelas, para que se especialicen (...) de manera que no haya ningún peligro en plantear y aumentar la productividad con la racionalización de los sistemas y en los lugares en que sea preciso con la instalación de nuevas maquinarias... (Guevara, 1966b, p. 153)

Y, sobre este mismo tópico, añadió las siguientes ideas:

La solución de la contradicción entre el avance técnico y el empleo debe resolverse de todos modos mediante el gasto productivo en un régimen de justicia social; nuestro planteamiento es que se valoricen estos gastos, dándole el énfasis a la preparación de cuadros técnicos. (Guevara, 1970, p. 25)

Sobre la base de estas ideas, el Che priorizó la creación y desarrollo de las Escuelas Populares, más de cincuenta en diferentes provincias del país, para la capacitación y entrenamiento de los cinco mil obreros excedentes de la industria del calzado sin afectación de sus salarios para elevar su escolaridad hasta el término del nivel primario y después calificarse en los distintos oficios necesarios a los distintos talleres e industrias mecánicas de las diferentes provincias. Esta fue una tarea que requirió de grandes esfuerzos organizativos, obteniéndose resultados positivos en el aporte de fuerza cualificada para la naciente industria mecánica y en la mejoría de la productividad y eficiencia de la industria del calzado. 
El Che priorizó tres tareas en 1964: impulsar la capacitación masiva de los trabajadores en coordinación con las empresas y organismos; asegurar la capacitación técnica para las nuevas unidades y confeccionar un plan perspectivo de capacitación. Estas mismas tareas se repitieron para 1965, añadiéndose otras de mayor alcance: la coordinación con las universidades, con el objetivo de conseguir una adecuada formación de personal técnico universitario conforme las necesidades del organismo; así como la organización de cursos por correspondencia para administradores y funcionarios administrativos.

Para poder valorar el esfuerzo realizado, solamente es necesario mencionar que entre 1963 y 1964 se graduaron, en los diferentes cursos de capacitación masiva, alrededor de dieciséis mil trabajadores en todo el país, y, entre 1964 y 1965, cerca de dos mil para las diferentes nuevas plantas en construcción (Viceministerio para el Desarrollo Técnico, 1964, p. 213).

La Dirección tuvo la responsabilidad de organizar y dirigir varias escuelas. Una de las primeras y de las que recibió una atención muy directa del Che, fue la Escuela de Administradores. Su misión era dotar al personal seleccionado de un nivel básico de enseñanza primaria -no se podía esperar más en aquellos momentos- y elementos de planificación, de contabilidad y otras materias necesarias para dirigir una fábrica. Fueron matriculados alrededor de doscientos alumnos. La escuela se nutrió, entre otros, de muchos de aquellos administradores «improvisados», quienes tuvieron que asumir funciones gerenciales en los momentos difíciles de la nacionalización de industrias. De esa escuela salieron formados muchos compañeros quienes asumieron posteriormente, con éxito, cargos de mayor responsabilidad.

La Escuela de Dibujantes Mecánicos, que recibió inicialmente una colaboración soviética, daba soporte a la naciente industria de esa rama. En diciembre de 1963 se graduaron los primeros ciento cincuenta alumnos. La Escuela de Superación Administrativa preparaba personal para ejercer funciones tales como jefes de personal, de compras, de almacenes, etc. en fábricas y empresas. En fin: las valoraciones realizadas del trabajo de la Dirección de Capacitación, por lo general, fueron positivas. En las conclusiones del informe presentado por la Dirección al Consejo de Dirección del Ministerio (1963), el Che expresó que se hacían proposiciones y se ofrecían soluciones que demostraban capacidad por resolver problemas; además, se detectaba un salto de calidad notable. Para 1964, volvió a observar, nuevamente, el salto de calidad que se alcanzaba.

Desde los primeros años de la Revolución, el esfuerzo por elevar el nivel cultural y educacional de todo el pueblo fue una tarea titánica. Ejemplo cimero fue la Campaña de Alfabetización, cumplida en un año con la participación masiva de millares de alfabetizadores, en su mayoría jóvenes brigadistas ${ }^{2}$. Los momentos eran extremadamente difíciles: los alzados contrarrevolucionarios principalmente en la Sierra del Escambray y en muchos lugares del país (financiados por la $\mathrm{ClA}$ ), el ataque mercenario por Playa Girón, otros actos de sabotaje y los viles asesinatos de alfabetizadores y campesinos siendo alfabetizados.

(2) Las brigadas «Conrado Benítez», conformadas por jóvenes estudiantes, obreros y profesores de manera voluntaria, quienes además de alfabetizar participaban en las labores productivas de los campesinos en las zonas rurales. 
El Ministerio de Educación, que en aquella época también asumía las actividades de la educación superior, tenía grandes tareas que cumplir. Aún a pasos de gigante, era imposible que ese Ministerio tratara de marchar sólo para alcanzar sus metas. La educación se convirtió para el MININD en tarea de todos. Por ello, fue necesario que éste abordara muchas de las tareas de capacitación anteriormente explicadas. Hubiera sido imposible delegar en esos momentos la responsabilidad al Ministerio de Educación de atender tantas actividades.

El Che siguió un criterio muy utilizado por él: lo que él llamaba el método de la «tracción paralela», o sea, impulsar tareas que, aunque estrictamente no le correspondieran, eran importantes para el país.

\section{La experiencia de la Escuela Vladimir Ilich Lenin}

Al terminar la Campaña de Alfabetización, el Che recibió una carta de un joven recién alfabetizado, la que, independientemente de inevitables faltas de ortografía y de sintaxis, mostraba la presencia de una gran inteligencia. Esa carta la leyó en un Consejo de Dirección, preguntándose sobre cuántas personas jóvenes estarían por distintos lugares, con un alto nivel de inteligencia, que hasta ahora no tuvieron la posibilidad de estudiar; que tienen espíritu revolucionario y están dispuestas a realizar sacrificios para poder superarse. Si les creásemos determinadas condiciones, estos jóvenes podrían «quemar etapas»-idea siempre presente en el pensamiento y acción del Che- y llegar a ser, en tiempo breve, graduados universitarios.

Sobre la base de estas ideas, se elaboró un embrión de programa que se podría sintetizar en los siguientes aspectos:

a) Realizar un trabajo de búsqueda por todo el país, apoyándonos en nuestras empresas y delegaciones provinciales, para localizar jóvenes brillantes, con baja escolaridad, dispuestos, en un período de dos o tres años, a terminar la enseñanza media para ingresar en la universidad.

b) Seleccionar una escuela donde estos jóvenes pudieran internarse durante todo ese tiempo.

c) Elaborar un programa de estudio para en ese breve período de tiempo, logren culminar la enseñanza media. La formación universitaria se realizaría en las distintas universidades.

d) Seleccionar un cuerpo adecuado de profesores.

Sobre esta base, la Dirección de Capacitación dio los pasos necesarios para iniciar las labores de esta escuela, con alumnos y profesores seleccionados de diferentes provincias del país.

Varios especialistas del Ministerio de Educación expresaron que el método seguido era antipedagógico. El Che insistió en que no se trataba de una escuela del Sistema Nacional de Educación; con normas y parámetros establecidos para cursos regulares. Esta era una escuela especial para alumnos también especiales. El programa, por tanto, estaba diseñado para personas que mostraran un alto nivel de inteligencia y disposición para sacrificarse y «quemar etapas». 
En la asamblea inicial celebrada con los alumnos seleccionados, el Che les explicó con toda claridad, el esfuerzo que de ellos se iba a requerir. Además de estudiar mucho, estarían separados de la familia -aunque periódicamente tendrían salidas para visitarlas- estarían fuera de su medio ambiente habitual, etc. En aquel momento, varios alumnos no parecieron convencidos $y$, por ende, algunos causaron baja después.

Más de veinte años después, fui convidado a dar una conferencia sobre el Che en el entonces Ministerio de la Industria Básica (actualmente Ministerio de Energía y Minas) y, entre varias cuestiones, mencioné la creación de esta escuela, indicando que yo no conocía cuales habían sido los resultados de ese experimento ya que me habían designado para asumir un papel dirigente en otra institución. Dos manos se levantaron de entre el público. Eran dos ingenieros que se habían preparado en la mencionada Escuela.

\section{La Dirección de Colaboración Científico-Técnica}

Esta Dirección, también adscrita al Viceministerio para el Desarrollo Técnico, tenía, entre sus funciones, la obtención de asistencia técnica extranjera para vincularla a la selección y envío de becarios procedentes del conjunto de empresas y fábricas del ministerio para capacitarse en el exterior. Era, por tanto, una importante fuente de obtención y preparación de recursos humanos calificados, así como la organización de la información científica y técnica.

Llegados este punto, es importante destacar que el ofrecimiento del gran número de becas que se renovaba anualmente por parte de la URSS y demás países del campo socialista mostraba un gran sentido solidario. Los becarios recibían para su manutención personal, alimentación, vivienda y una modesta cantidad de dinero para sus gastos personales. La parte cubana se responsabilizaba con mantener el salario de cada uno para el sustento de su familia.

El Che consideraba que el trabajo tecnológico mediante la ayuda de los países amigos para elevar la calificación de nuestros especialistas y técnicos, particularmente en las nuevas ramas partiendo de la base de nuestras experiencias, así como en los sectores más tradicionales a los que les falta suficiente personal calificado contribuirá a sentar las bases para un desarrollo independiente. En ese sentido, orientó garantizar una atención eficiente a la asistencia técnica extranjera que se recibía, así como ejercer un control sistemático y efectivo para garantizar una adecuada utilización de sus servicios. Determinó, pues, que se realizase un control eficiente de los becarios que, en números cada vez creciente, salían a los países socialistas. También indicó mejorar e impulsar la colaboración con las universidades y trabajar en estrecho contacto con los institutos y empresas, para actuar dinámicamente en la solución de sus problemas a través de la colaboración. Dicho de otra manera: el concepto de colaboración se ampliaba para la cooperación nacional.

El volumen de trabajo de esta Dirección era considerable. En aquellos primeros años no existía todavía una Comisión Nacional para la Colaboración Científico-Técnica, inaugurada en 1964, con la misión de regular y controlar, a nivel nacional, estos aspectos. Por otra parte, no existía capacidad suficiente en el Sistema Nacional de Educación para preparar todos los obreros calificados y técnicos medios necesarios para el país. Se estipuló que cada organismo se encargara de la selección, atención y evaluación de sus 
becarios en el extranjero. Esto sería una función de los Ministerios de Educación y de Educación Superior posteriormente.

Así, subrayamos que, entre diciembre de 1963 y junio de 1964, trabajaban 635 especialistas extranjeros en todo el MININD, incluyendo sus empresas, fábricas e institutos; 107 practicantes se especializaban en el extranjero para las nuevas fábricas; 1201 eran becarios de todo tipo, 774 de los cuales estudiaban carreras universitarias, 109 técnicos medios y 318 obreros calificados. (Viceministerio de Desarrollo Técnico, 1964, p. 231)

Dos estudiantes, en 1964, fueron enviados a la URSS para estudiar Física Nuclear. Cuando yo vi esto en el Plan de Becas, me pareció que era innecesario en aquellos momentos, en que nuestra preocupación era construir plantas termoeléctricas de sólo 100 MW. La energía nuclear me parecía algo de ciencia-ficción en esos momentos. Pregunté y me informaron que eran orientaciones del Che. Entonces le pregunté a él directamente. Me respondió: «El futuro de la energía parece ser nuclear. El futuro puede que esté lejos, pero el futuro hay que comenzarlo a construir desde ahora.»

\section{La atención a los becarios}

El número de becarios en diversos países socialistas, la heterogeneidad de sus niveles educacionales; el hecho de que una gran mayoría nunca había viajado al extranjero y, por otra parte, el choque de hallarse en países de diferentes idiomas, diferentes culturas y tradiciones requería de una especial atención.

El Che, en una reunión con ellos, les advirtió de estas circunstancias y enfatizó la necesidad de que ellos aprovecharan al máximo la oportunidad y responsabilidad que se les brindaba; que ellos eran una muestra de la calidad humana y revolucionaria del pueblo cubano y que, si por negligencia o problemas graves de conducta, perdían la beca, eso representaba una mancha para esa imagen que ellos deberían portar y que, por otra parte, le habían quitado a otro compañero la oportunidad de haber ganado esa beca; por tanto, ante esa situación, deberían ser sancionados administrativamente.

\section{Consideraciones finales}

En el pensamiento y en las acciones tomadas por el Che como Ministro de Industrias, es evidente que el hombre aparece como centro de su concepción integral sobre cómo desarrollar la nueva sociedad y, muy en particular, el desarrollo del hombre en el socialismo. Por eso es necesario insistir en su concepción para que la humanidad progresista $y$, en particular, las nuevas generaciones, vean en su figura la encarnación de altísimos valores éticos y morales de solidaridad, generosidad, austeridad, ejemplo personal, sensibilidad humana, decisión y disposición de ofrecer hasta la propia vida por la liberación de los pueblos.

Fidel, en la velada solemne en memoria del Che, celebrada en la Plaza de la Revolución, pocos días después de su muerte, el 18 de octubre de 1967, expresó:

Cuando pensamos en su vida, cuando pensamos en su conducta, que constituyó el caso singular de un hombre rarísimo en cuanto fue capaz de conjugar en su personalidad no solo las características de hombre de acción, sino también de hombre de pensamiento, de hombre de inmaculadas virtudes revolucionarias y de extraordinaria sensibilidad humana, unidas a un carácter de hierro, a una voluntad de acero, a una tenacidad indomable. (Castro, 1967) 


\section{Referencias}

Castro, F. (1967) «Discurso en la velada solemne en memoria del Comandante Ernesto Che Guevara en la Plaza de la Revolución, La Habana, 18 de octubre». Granma, 19 de octubre de 1967, p. 3.

Fernández, J. R. (1975) «Palabras pronunciadas en la clausura del iv Seminario Juvenil de Estudios Martianos». Educación, vol. 5, número 16, pp. 183-206.

Guevara E. (1962) «Tareas industriales de la revolución en los años venideros», Cuba Socialista, 2(7), pp. 20-45.

Guevara E. (1966a) «Discurso en una reunión con los directores y jefes de capacitación de las Empresas Consolidadas y secretarios de Educación y Trabajo de los veinticinco sindicatos nacionales de industrias, 16 de marzo, 1962», en El Che en la Revolución Cubana. Tomo 6. La Habana, Ministerio del Azúcar, pp. 151-162.

Guevara E. (1966b) «Reuniones bimestrales, 20 de enero de 1962», en El Che en la Revolución Cubana. Tomo 6. La Habana, Ministerio del Azúcar, pp. 145-172.

Guevara, E (1970) Una actitud nueva ante el trabajo. Obras. 1957-1967. La Habana, Casa de las Américas.

Guevara E. (1972) Discusión colectiva y responsabilidades únicas. Escritos y discursos. La Habana, Editora Política.

Guevara E. (1977) Escritos y discursos. La Habana, Editorial de Ciencias Sociales.

Guevara E. (2001) Che Guevara habla a la juventud. New York, Pathfinder.

Viceministerio para el Desarrollo Técnico (1964) Informe al Consejo de Dirección. La Habana, Mimeo. 


\section{El Che Guevara i el desenvolupament dels recursos humans a Cuba}

Resum: A la vida i obra d'Ernesto Che Guevara (1928-1967) hi ha aspectes la indagació dels quals ha de contribuir, per ser menys coneguts, a ressaltar el seu destacat paper en la construcció del socialisme a Cuba, des de la seva posició com a Ministre d'Indústries. En aquesta funció va exercir un important paper en el procés d'industrialització cubà i, vinculat al mateix, en la promoció i el desenvolupament dels recursos humans en aquest àmbit. Tot plegat es sustentava en un profund desenrotllament de la consciència de l'ésser humà i del paper d'aquest com a actor central i objectiu principal en la construcció de la nova societat socialista. En el present article es mostren les concepcions, les prioritats establertes i les accions conseqüents preses pel Che en aquest sentit.

Paraules clau: Che Guevara, recursos humans, capacitació, socialisme, revolució cubana, Ministeri d'Indústries de Cuba

\section{Le Che Guevara et le développement des ressources humaines à Cuba}

Résumé: Dans la vie et l'œuvre d'Ernesto Che Guevara (1928-1967), on trouve des aspects moins connus et dont l'étude doit contribuer, de ce fait, à mettre en avant son rôle de premier plan dans la construction du socialisme à Cuba, depuis sa position en tant que ministre de l'industrie. Dans le cadre de cette fonction, il a joué un rôle important dans le processus d'industrialisation cubain et, en lien avec celui-ci, dans la promotion et le développement des ressources humaines dans ce domaine. Tout ceci s'appuyait sur un développement profond de la conscience de l'homme et du rôle de ce dernier en tant qu'acteur central et objectif principal dans la construction de la nouvelle société socialiste. Le présent article montre les conceptions, les priorités établies et les actions conséquentes prises par le Che à cet égard.

Mots-clés: Che Guevara, ressources humaines, formation, socialisme, révolution cubaine, ministère de l'industrie cubain

\section{Che Guevara and the development of human resources in Cuba}

Abstract: In the life and work of Ernesto Che Guevara (1928-1967) there are certain little known aspects whose investigation will highlight his prominent role in the construction of socialism in Cuba, from his position as Minister of Industry. In this position, he played an important role in the Cuban industrialization process and, as a result, in the promotion and development of human resources in this area. All this was based on a profound development of man's consciousness and his role as a central actor and the main objective in the construction of the new socialist society. This article shows the conceptions, the established priorities, and the actions taken by Che in this regard.

Keywords: Che Guevara, human resources, training, socialism, Cuban revolution, Ministry of Industries of Cuba. 